EPJ Web of Conferences 82, 01003 (2015)

DOI: $10.1051 /$ epjconf/20158201003

(C) Owned by the authors, published by EDP Sciences, 2015

\title{
Choice of the sizes of samples in case of determination of heat diffusivity of optical quartz glass by method of laser pulse
}

\author{
Mark Katz ${ }^{\mathrm{a}}$ \\ Ministry of Education and Science of the Russian Federation the National Research Tomsk Polytechnic \\ University Tomsk, Russia
}

\begin{abstract}
The analysis of errors in the determination of thermal diffusivity of optical quartz glass, caused by radiative energy transfer in the heated layer of material in the corresponding implementation of the method of the laser pulse when exposed to the surface of the collimated laser pulse of finite duration. Influence on value of this error of the geometrical sizes of a sample and process of absorption of radiation is set.
\end{abstract}

\section{Introduction}

Now one of the methods which are widely applied to determination of thermal diffusivity of materials in the moderate range of temperatures is the method of laser pulse [1,2]. It is based on the absorption in a thin layer in the front "hot" surface of the sample pulse of radiant energy and an approximate solution of a one-dimensional heat conduction problem for an infinite plate.

The maximum temperature of overheating of $T_{\max }$, time $\tau_{05}$ achievements of a half of maximum temperature of overheating is determined by the received heat pattern of the reverse "cold" (not heated) surface of a sample. thermal diffusivity of $a$, specific heat capacity of $c$ and heat conduction of material $\lambda$ on formulas [1] are calculated.

Practical interest represents use of this method for determination of thermal diffusivity of the optical quartz glass which is characterized from the used modifications of glasses by the limit characteristics: high temperature of emolliating and evaporation, low coefficient of thermal extension, high optical transparency and chemical firmness [3]. Process of heating of such materials is followed by accumulation of the warmth which arrived not only due to heat conduction, but also and volume absorption in the certain areas of a range depending on wavelength of thermal radiation.

Conditions of application of a method of laser pulse for determination of heat diffusivity of the partially transparent materials are considered in [4-6] within one-dimensional tasks of heat conduction. It is set [4-6] that in case of determination of heat diffusivity growth of the methodical errors caused by intensification of process of heating due to radiation heat transfer in a sample in comparison with set earlier [7] for opaque materials is possible.

\footnotetext{
${ }^{a}$ Corresponding author: katz@tpu.ru
}

This is an Open Access article distributed under the terms of the Creative Commons Attribution License 4.0, which permits unrestricted use, distribution, and reproduction in any medium, provided the original work is properly cited. 


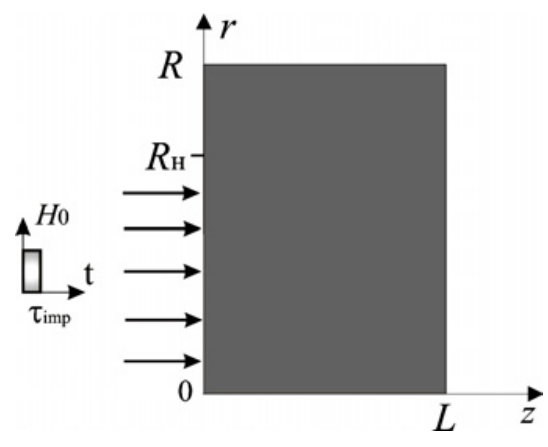

Figure 1. Scheme of solutions domain.

It is shown [4-6] that in case of practical implementation of a method of laser pulse restrictions on lengths of waves of the falling radiation and absorption coefficients are necessary.

However the model of heat conduction [1] in many cases doesn't consider mismatch of the cross sizes of the samples and sections of a surface heated to the course of short pulse of laser radiation. As a rule, it is connected to features of structure of the researched material and difficulties of manufacture of samples of the small sizes. In this regard the assessment of errors of determination of heat diffusivity of translucent materials by method of laser pulse in conditions of mismatch of the cross sizes of a sample to the cross sizes of the section of its surface heated by a laser radiation is expedient. In this operation the assessment of an error of determination of heat diffusivity of optical quartz glass by method of laser pulse [1] within model of conductive and radiation heat transfer in a sample is considered in case of the cross sizes of the heated sections of surfaces, the smaller cross sizes of a sample.

\section{Statement of the problem}

In case of a problem definition it was supposed that material is gray, and own radiation isn't enough in comparison with an external radiation flow.

Such assumption doesn't superimpose essential restrictions on a community of setting of the task and real conditions of a heat transfer through translucent material.

As a rule, for carrying out experiments by a technique [1] samples are made in the form of disks. The transverse section of a laser beam represents a circle. For an assessment of scales of influence of processes of radiation heat transfer in a heated-up layer of material the two-dimensional task of heat conduction for a disk of the limited sizes in case of impact on a section of its surface of the collimated laser radiation of temporal duration and absence of heat exchange on a section is solved "hot" and all "cold" boundaries (Fig. 1) which aren't heated by laser pulse.

Possible effects of change of values of heatphysical characteristics with change of temperature weren't considered. Such assumption is quite justified for many materials in the real range of possible change of temperatures in experiment process even in case of high values of specific heat fluxes to the heated sample surface in connection with a small pulse duration. The area of the decision represented a cylindrical disk of small thickness of $L$ and the radius of $R$ (Fig. 1) which section of boundary heated up the collimated energy flow directed perpendicular to the plane of the heated surface in case of radiant density of $H_{0}$ and a pulse duration $\tau_{\text {imp. }}$. Radius of a transverse section of a laser beam of $R_{\mathrm{H}}$.

The mathematical model corresponding to the accepted problem definition (Fig. 1) includes a nonstationary differential equation of energy in private derivatives (1) with boundary (2-7) and initial 
(8) conditions:

$$
\begin{gathered}
c \rho \frac{\partial T}{\partial t}=\frac{\lambda}{r} \cdot \frac{\partial}{\partial r}\left(r \frac{\partial T}{\partial r}\right)+\lambda \frac{\partial^{2} T}{\partial z^{2}}-\frac{d H(z)}{d z}-\frac{d H(r)}{d r}, \quad 0<z<L ; 0<r<R \\
z=0: \quad-\lambda\left(\frac{\partial T}{\partial z}\right)=H_{0}, \quad r \leq R_{\mathrm{H}}, 0<t \leq \tau_{\mathrm{imp}} ; \\
z=0: \quad-\lambda\left(\frac{\partial T}{\partial z}\right)=0, \quad R>r>R_{\mathrm{H}}, t>0 ; \\
z=0: \quad-\lambda\left(\frac{\partial T}{\partial z}\right)=0, \quad t>\tau_{\mathrm{imp}} ; \\
z=L: \quad-\lambda\left(\frac{\partial T}{\partial z}\right)=0, \quad 0<r<R, \quad t>0 ; \\
r=0: \quad-\lambda\left(\frac{\partial T}{\partial r}\right)=0, \quad 0<z<L, t>0 ; \\
r=R: \quad-\lambda\left(\frac{\partial T}{\partial r}\right)=0, \quad 0<z<L, t>0 ; \\
t=0 ; \quad T=T_{0} .
\end{gathered}
$$

where $\rho$-density of material; $T, T_{0}$ - the current and starting value of temperature; $t$-time; $H(z), H(r)$ - density of a radiant flux in material in the coordinate directions $\mathrm{z}$ and $\mathrm{r}$.

Changing the energy density of radiation-tion of the material was determined by the BouguerLambert-Beer [8]:

$$
\begin{aligned}
& H(z)=H_{0} \cdot \exp \left(-k_{\lambda} \cdot z\right), \\
& H(r)=H(z) \cdot \exp \left(-k_{\lambda} \cdot r\right),
\end{aligned}
$$

where $k_{\lambda}$ - absorption coefficient of the collimated laser radiation flow, $\mathrm{m}^{-1}$.

\section{Solution method}

Boundary value problem (1)-(8) is solved by finite difference method using the iterative algorithm $[9,10]$, developed for the solution of nonlinear time-dependent problems of high-temperature heat transfer in areas with locally concentrated sources of energy. The difference analogs of a differential equation (1) and appropriate edge conditions are solved by a local and one-dimensional method [11].

\section{Results and discussion}

Numerical modeling of process of a heat transfer is carried out on the example of optical quartz KU1 glass with thermal characteristics: $\left.\lambda=1,35 \mathrm{~W} /(\mathrm{m} \cdot \mathrm{K}), c=728 \mathrm{~J} /(\mathrm{kg} \cdot \mathrm{K}), \rho=2200 \mathrm{~kg} / \mathrm{m}^{3}\right)$ [12]. Thickness of a sample changed from 1 to $3 \mathrm{~mm}$ with change of its radius $8,10,12 \mathrm{~mm}$.

The wavelength range of the transparency region of the vitreous silica is $2100 \ldots 2800 \mathrm{~nm}$, which corresponded to a change in the absorption coefficient from 10 to $250 \mathrm{~m}^{-1}$ [12]). Radius of a circle of laser heating of $R_{\mathrm{H}}$ in case of numerical modeling made $80 \%$ of radius of a sample for an exception 


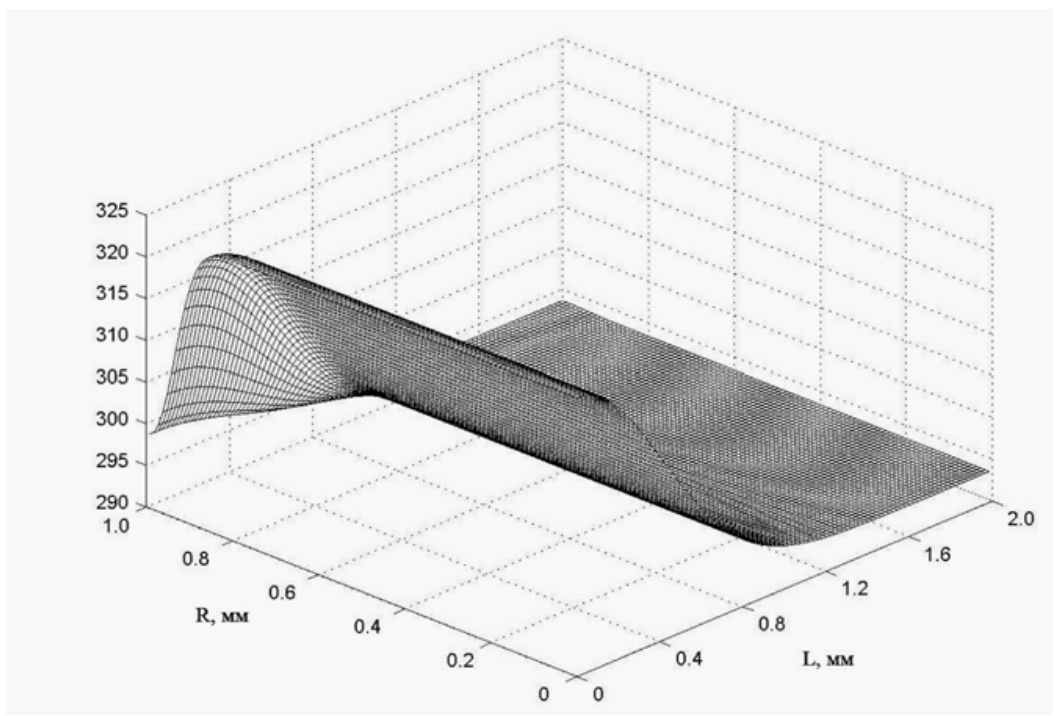

Figure 2. Distribution of $T(z, r)$ in a sample $(R=10 \mathrm{~mm}, L=2 \mathrm{~mm})$ at time $t=0,5 \mathrm{~s}$.

of edge effects of interaction of a laser radiation with an external isolation layer [7]. The choice of values radiative heat flow $H_{0}=5 \cdot 10^{7} \mathrm{~W} / \mathrm{m}^{2}$ and heating duration $\tau_{\text {imp }}=0,5 \mathrm{~ms}$ performed taking into account the basic methods of [1] - obtaining "overheat temperature" to "cold" surface of a sample of at least $3 \ldots 6^{\circ} \mathrm{C}$.

In Fig. 2 distribution of $T(z, r)$ is given in a sample $(R=10 \mathrm{~mm}, L=2 \mathrm{~mm})$ at time $t=0,5 \mathrm{~s}$.

It is set (Fig. 2) that in case of a local uniform heat flux in a heating zone temperature changes not only on disk thickness, but also on radial coordinate. It leads to change of value in comparison with a one-dimensional problem definition [1]. The obtained results allow us to conclude much the multidimensional distribution of heat in the system under study and its impact on the error in the determination of thermal diffusivity of the material. Figure 3 shows the calculated after solving the problem (1-8) depending on thermal error $\left(\delta_{a}\right)$ optical quartz glass of the thickness $L$ of the sample at an absorption coefficient $k_{\lambda}=20 \mathrm{~m}^{-1}, \mathrm{k}_{\lambda}=100 \mathrm{~m}^{-1}$ and the radii of heated area, my sample surface 8 , $10,12 \mathrm{~mm}$. By results of numerical simulation it is set that values depend on thickness of a sample and absorption coefficient of material. In case of $k_{\lambda}=20 \mathrm{~m}^{-1}$ (Fig. 3a) and an $L=2 \mathrm{~mm}$ error $\delta_{a} 8 \%$ aren't exceeded. In case of $k_{\lambda}=100 \mathrm{~m}^{-1}$ value $\delta_{a}$ (Fig. 3b) and the similar sizes of a sample of an error made about $18 \%$.

With increase in thickness of a sample of value increase and reach the maximum value in case of $L=3 \mathrm{~mm}(R=10 \mathrm{~mm})$ in case of values of $k_{\lambda} 20 \mathrm{~m}^{-1}$ and $100 \mathrm{~m}^{-1}$ respectively about $6 \%$ and $15 \%$. Rather significant change of value is caused by heattransfer on the radial coordinate of $r$ during the period after extinction of laser influence.

Accumulated during the pulse in a very thin heated to a high temperature layer of the sample energy during this period redistributed by conduction and radiation coordinate direction $z$, in which the heat transfer is then used for the calculation as described in [1], thermal diffusivity, but also in the direction of $r$. Accordingly, the temperature $T_{\max }$ in the "cold" boundary will be quite significantly different from its counterpart in the case of one-dimensional heat conduction equation with the size of the "laser spot" and the radius of the sample material.

The main reason for the error $\delta_{a}$ is the mismatch of the transverse dimensions. The greater the radius of the sample will vary and the heating region (see Fig. 3), the $\delta_{a}$ greater becomes. The same values 
a)

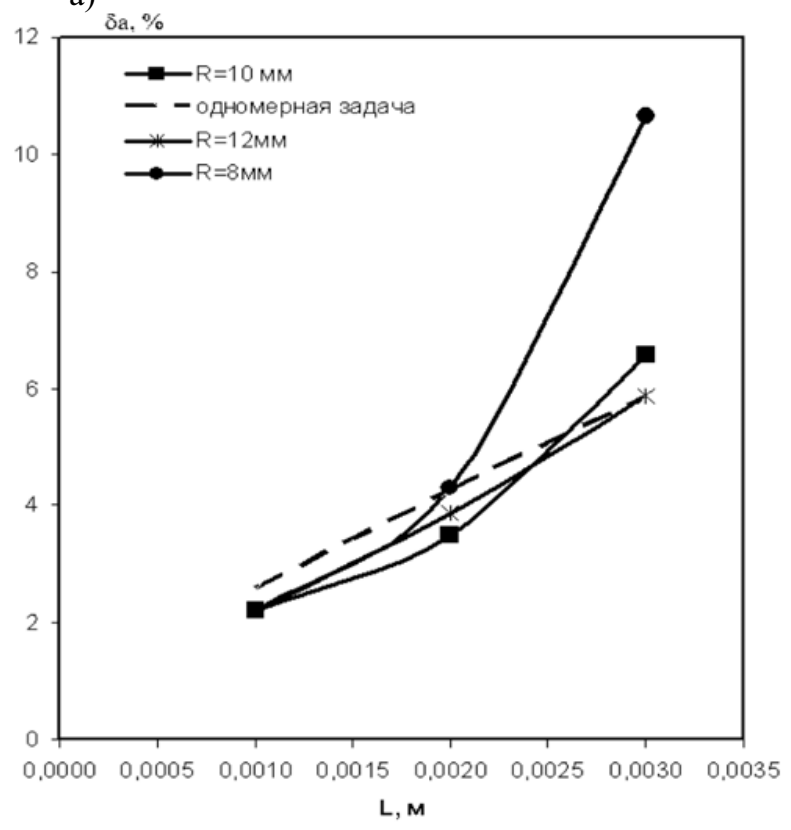

b)

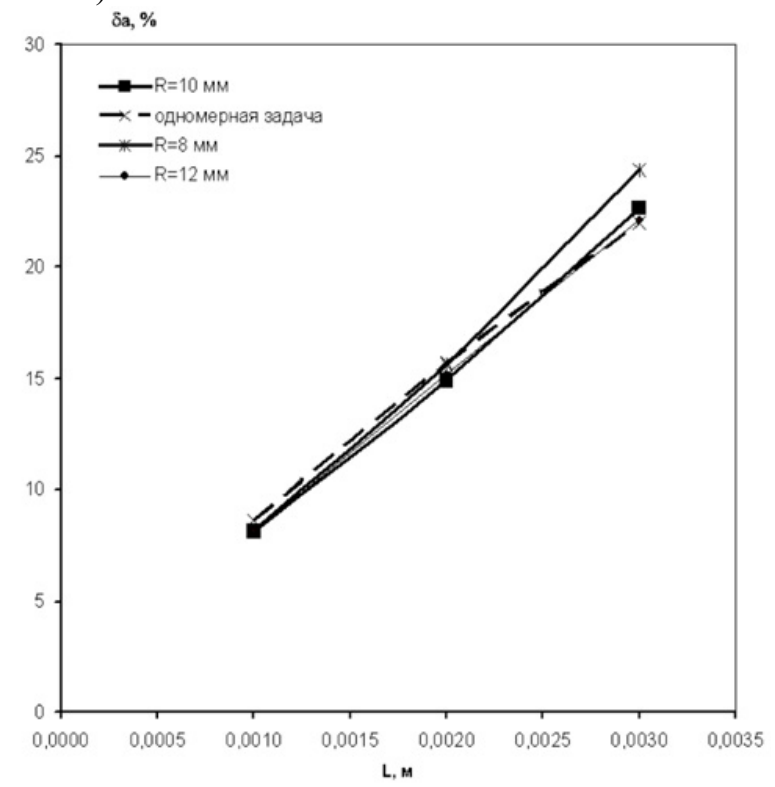

Figure 3. Dependences of an error of heat diffusivity $\delta$ a optical quartz glass from thickness of a sample of $L$ in case of absorption coefficient $k_{\lambda}$ : a) $20 \mathrm{~m}^{-1}$; b) $100 \mathrm{~m}^{-1}$.

of $L$ error in the determination of thermal diffusivity is smaller, the larger the radius $R$ of the heated portion of the surface of the sample.

With the growth of $R$ (all other conditions remaining the same) two-dimensional nature of the heat transfer will be shown at the point of measurement (on the symmetry axis of the sample) to a lesser extent. 
Analysis of the results (Fig. 3) suggests the existence of minimum providing methodological errors in determining the thermophysical properties of translucent materials, the thickness of the samples at other appropriate experimental conditions.

By selecting the thicknesses of the samples can be reduced to a minimum value of systematic errors determination of thermal (and, accordingly, other thermophysical characteristics) translucent materials.

\section{References}

[1] W.J. Parker, R.J. Jenkins, C.P. Butler, J. Appl. Phys. V. 32, 9 (1961)

[2] O. Altun, B. Erhan, A. Kalemtas, J. Achievements in Materials and Manufacturing Engineering V 302 (2008)

[3] L.A. Novitsky, B.M. Stepanov, Optical properties of materials at low temperatures (Mechanica energetika, Moscow, 1980)

[4] G.V. Kuznetsov, M.D. Katz, Izmeritelnaja technica 9 (2012)

[5] G.V. Kuznetsov, M.D. Katz, News of the St.-Petersburg state institute of technology (technical university. 14 (2012)

[6] M.D. Katz EPJ Web of Converences 76010017 (2014) URL: http://dx .doi.org/10.1051/ epjconf /20147601017

[7] G.V. Kuznetsov, M.D. Kats, Measurement Techniques 52 I 4 (2009)

[8] B.M. Yavorsky, A.A. Detlaf, The reference manual on physics (Nauka, Moscow, 1979)

[9] G.V. Kuznetsov, M.A. Sheremet, Thermophysics and Aerodynamics 16. 1 (2009)

[10] G.V. Kuznetsov, M.A. Sheremet, Russian Microelectronics 37 (2008)

[11] A.A. Samarskiy, Theory of the difference schemes (Nauka, Moscow 1983)

[12] I.K. Kikoin, Tables of physical values: reference manual (Atomizdat, Moscow, 1976) 